\title{
Infrared spectroscopy of NGC 1068: Probing the obscured ionizing AGN continuum
}

\author{
Tal Alexander ${ }^{1}$ \\ Dieter Lutz ${ }^{2}$, Eckhard Sturm ${ }^{2}$, Reinhard Genzel ${ }^{2}$ \\ Amiel Sternberg ${ }^{3}$, Hagai Netzer ${ }^{3}$
}

\begin{abstract}
The ISO-SWS 2.5-45 $\mu \mathrm{m}$ infrared spectroscopic observations of the nucleus of the Seyfert 2 galaxy NGC 1068 (see companion paper) are combined with a compilation of UV to IR narrow emission line data to determine the spectral energy distribution (SED) of the obscured extreme-UV continuum that photoionizes the narrow line emitting gas in the active galactic nucleus. We search a large grid of gas cloud models and SEDs for the combination that best reproduces the observed line fluxes and NLR geometry. Our best fit model reproduces the observed line fluxes to better than a factor of 2 on average and is in general agreement with the observed NLR geometry. It has two gas components that are consistent with a clumpy distribution of dense outflowing gas in the center and a more extended distribution of less dense and more clumpy gas farther out that has no net outflow. The best fit SED has a deep trough at $\sim 4$ Ryd, which is consistent with an intrinsic Big Blue Bump that is partially absorbed by $\sim 6 \times 10^{19} \mathrm{~cm}^{-2}$ of neutral hydrogen interior to the NLR.
\end{abstract}

\section{Introduction}

The intrinsic spectral energy distribution (SED) of active galactic nuclei (AGN), which extends from the radio up to $\gamma$-rays, cannot be directly observed from the Lyman limit and up to several hundred eV due to Galactic and intrinsic absorption. However, the extreme-UV (EUV) and soft X-ray continuum can be investigated indirectly by the infrared coronal line emission. These lines are emitted by collisionally excited forbidden fine-structure transitions of highly ionized atoms, whose ionization potentials extend well beyond the Lyman limit up to hundreds of

\footnotetext{
${ }^{1}$ Institute for Advanced Study, Olden Lane, Princeton, NJ 08540,USA

${ }^{2}$ Max-Planck-Institut für Extraterrestrische Physik, Postfach 1603, D-85740 Garching, Germany

${ }^{3}$ School of Physics and Astronomy and Wise Observatory, Raymond and Beverly Sackler Faculty of Exact Sciences, Tel Aviv University, Ramat Aviv, Tel Aviv 69978, Israel

${ }^{4}$ Based on observations made with ISO, an ESA project with instruments funded by ESA member states (especially the PI countries: France, Germany, The Netherlands and the United Kingdom) and with the participation of ISAS and NASA. The SWS is a joint project of SRON and MPE.
} 
$\mathrm{eV}$. Unlike the strong permitted lines of these ions, which are also emitted in the obscured EUV, the reddening-insensitive forbidden IR coronal lines and semi-forbidden optical coronal lines can be observed. Therefore, when photoionization is the main ionization mechanism, the coronal lines can provide information on the intrinsic obscured SED and the accretion process that powers the AGN. This information can be extracted by photoionization models of the NLR.

The coronal lines are collisionally suppressed in the dense broad line region (BLR) close to the continuum source and are efficiently emitted only from the more rarefied gas in the narrow line region (NLR), hundreds of pc away from the center. It is well established that large quantities of gas attenuate the continuum emission in many AGN. These gas clouds are detected by narrow UV absorption lines (e.g. Kriss et al. 1992a) or by X-ray absorption features and emission lines (e.g. George et al. 1998). Although their exact location along the line of sight is unknown, there are reasons to believe that in some cases they may be inside the NLR. In particular, the warm absorbers that block the X-ray continuum appear to cover a large fraction of the continuum source (George et al. 1998). This raises the possibility that in some AGN the ionizing SED, which is traced by the coronal lines, is not the intrinsic one produced by the accretion process, but rather one that is filtered by intervening absorbers inside the NLR. It has been proposed that such absorbers are common in Seyfert galaxies, and are responsible for the observed correlations between the soft X-ray slope and the narrow emission line spectra of Seyfert 1.5 galaxies (Kraemer, Ruiz \& Crenshaw 1999).

This study of the Seyfert 2 galaxy NGC 1068 is part of the ISO-SWS program on bright galactic nuclei. Previous studies in this program include the reconstruction of the SED of the Seyfert 2 Circinus galaxy (Moorwood et al. 1996; Alexander et al. 1999) and of the Seyfert 1 Galaxy NGC 4151 (Alexander et al. 1999). In both cases we found evidence of a "Big Blue Bump" signature of a thin accretion disk (Shakura \& Sunyaev 1973). However, in the case of NGC 4151 this structure is masked by a deep absorption trough of an absorber situated between the BLR and the NLR, which filters the light that photoionizes the NLR.

In this paper we apply our SED reconstruction method to NGC 1068, one of the closest, brightest and most extensively studied Seyfert 2 galaxies, which is considered a prototype of this AGN class. The first detection of broad permitted emission lines in the polarized light of NGC 1068 (Antonucci \& Miller 1985) provided a major argument for the Seyfert 1 and 2 unification scheme (Antonucci 1993). This scheme postulates that the two Seyfert types have both broad and narrow line regions and an obscuring torus that lies between the two. When the torus is face on and the BLR is directly observed, the AGN is classified as a Seyfert 1. When the BLR is obscured by the torus, the AGN is classified as a Seyfert 2, and the BLR can be observed only indirectly in scattered polarized light. The factors that determine the accretion properties and the ionizing SED of AGN are currently unknown. However, the Seyfert unification picture implies that all possibly relevant factors being equal, such as luminosity, host galaxy type or redshift, the intrinsic SED of both AGN types should be similar. It is therefore of interest to complement our previous study of the nearby luminous Seyfert 1 galaxy NGC 4151 with a corresponding study of a nearby 
luminous Seyfert 2 galaxy with a similar host galaxy type, such as NGC 1068. The ISO-SWS observations of NGC 1068 are presented in a companion paper (Lutz et al. 2000) and are used there to derive the gas density and to place constraints on the structure and dynamics of the NLR.

This paper is organized as follows. In $\delta 2$ we summarize the physical properties of the nucleus of NGC 1068 that are needed for constructing the photoionization models and constraining their results. In $\$ 3$ we present the emission line flux compilation that we use in our modeling. In $\S$ we briefly discuss the construction and fitting of the NLR photoionization models. We present the results in $\$$ 国 and discuss them in $\$$.

\section{The physical properties of NGC 1068}

NGC 1068 is a barred spiral galaxy at $z=0.0036$ (distance $D=16.6 \mathrm{Mpc}$ for $H_{0}=65 \mathrm{~km} \mathrm{~s}^{-1} \mathrm{Mpc}^{-1}$ ) with magnitude $m_{B}=9.17$ (e.g. Lipovetsky, Neizvestny \& Neizvestnaya 1988). Observations of the nucleus of NGC 1068 and models of the nuclear line emission indicate that the gas in the nucleus forms a complex system, which is composed of various spatial and dynamical components. These are excited by several physical mechanisms, including photoionization by the nuclear continuum, photoionization by hot stars, and possibly also by shocks and energetic particles from a radio jet. In order to isolate the effects of the nuclear continuum and to construct photoionization models of the NLR it is necessary to understand the morphology and content of the galactic nucleus. We present here a brief overview of the properties of the nucleus that are relevant to this work.

\subsection{The galactic nucleus}

The most prominent morphological feature in the nucleus of NGC 1068 is the asymmetric bi-polar pattern of both the radio and the optical line emission. The radio emission extends over $\sim 15^{\prime \prime}$ and has a sharply defined northern lobe and a weaker diffuse southern lobe (Wilson \& Ulvestad 1983; Muxlow et al. 1996). The southern lobe is both smaller and redder, which is consistent with the picture that the large northern lobe is observed above the galactic disk, generally facing the observer, and the southern lobe is seen through the galactic disk (Unger et al. 1992; Macchetto et al. 1994). Images of the NLR in low excitation lines show mainly the northern

cone (Cecil, Bland \& Tully 1978; Unger et al. 1992; Evans et al. 1991). The precise value of the opening angle associated with the emission maps depends on the way the edge of the cone is defined and on the assumed position of the nucleus. The location of the nucleus can be determined to within $\sim 0.05^{\prime \prime}$ by the center of symmetry of the UV polarization pattern (Capetti et al. 1995, Kishimoto 1999). The position of the nucleus does not appear to coincide with the maximum of the continuum emission, which implies that the nucleus is heavily obscured even in the infrared. This is consistent with an obscuring torus of column density in excess of $10^{24} \mathrm{~cm}^{-2}$, as is inferred 
from X-ray (Marshall et al. 1993) and CO observations (Tacconi et al. 1994). The positioning of the nucleus makes it possible to estimate the opening angle of the radiation cone at $\gtrsim 70^{\circ}$ up to $\sim 100^{\circ}$. The ionization cone appears to be only partially filled.

Marconi et al. (1996) find that the coronal line emission peaks $0.5^{\prime \prime} \mathrm{NE}$ of the nucleus and extends up to $\sim 4^{\prime \prime}$. They note that the blueshift of the emission line profile centroid increases, and the FWHM of the profile decreases with $E_{\text {ion }}$ (the ionization energy required to produce the emitting ion from the preceding ionization stage). They interpret this as evidence that the emission lines are emitted from outflowing material. The high ionization lines are emitted in the inner light cone, where the velocity field is relatively coherent, while the lower ionization lines are emitted from slower, more extended areas with different velocities. The NLR [O III $] \lambda 5007$ emission extends over the few inner arcseconds (Evans et al. 1991; Unger et al. 1992; Dietrich \& Wagner 1998). The extended emission line region (EELR) [O III] $\lambda 5007$ emission extends over more than $10^{\prime \prime}$ (Unger et al. 1992).

The unresolved BLR is seen only in scattered polarized light and has FWHM of $\sim 3000$ $\mathrm{km} \mathrm{s}^{-1}$ (Miller et al 1990). As is seen in other Seyfert 2 galaxies (Wilson 1988; Capetti et al. 1996), the morphology of the NLR emission maps is correlated with that of the radio structure (Wilson \& Ulvestad 1983; Capetti et al. 1997). In particular, a 3-dimensional reconstruction of the positions of individual clouds based on polarimetric measurements also indicates that the northern NLR cone is directed towards the observer, and the southern part away from the observer (Kishimoto 1999). The close correspondence between the NLR and the jet suggests that the jet outflow sweeps and compresses the ambient gas and thereby increases the line emissivity. The NLR has a very complex structure (Macchetto et al. 1994) and displays large scale clumpiness. It is composed of many line emitting cloud complexes (Alloin et al. 1983; Meaburn \& Pedlar 1986; Evans et al. 1991; Dietrich \& Wagner 1998). The individual components have FWHM ranging from $\sim 200 \mathrm{~km} \mathrm{~s}^{-1}$ to $\sim 700 \mathrm{~km} \mathrm{~s}^{-1}$, and extend over $\sim 2500 \mathrm{~km} \mathrm{~s}^{-1}$ in velocity space, resulting in an integrated [O III] $\lambda 5007$ profile with FWHM of $1150 \mathrm{~km} \mathrm{~s}^{-1}$ (Dietrich \& Wagner 1998). The velocity field of the NLR clouds with the lowest FWHM is consistent with rotation around the nucleus. The bulk velocities of intermediate FWHM clouds are clearly split relative to the symmetry axis of the radio jet, which suggests that they are associated with the interaction between the radio jet and the NLR gas. The highest FWHM clouds are associated with highly polarized emitting structures (Capetti et al 1995), and could therefore be a reflected image of an inner obscured region. The EELR appears to follow the galactic rotation (Unger et al. 1992).

The large ISO apertures $\left(14^{\prime \prime} \times 20^{\prime \prime}\right.$ to $\left.20^{\prime \prime} \times 33^{\prime \prime}\right)$ includes both the outflowing inner NLR and the rotating EELR. 


\subsection{The line emitting gas}

There is evidence that at least three different ionization mechanisms are at work in the NLR. The very high ionization states are probably due to the the central continuum source. Marconi et al. (1996) find that the infrared coronal line ratios point to photoionization as the main excitation mechanism of the coronal gas, and that both collisional excitation or photoionization by very hot stars can be ruled out. At lower ionization states, the jet / ISM interaction can provide internal sources of excitation in addition to the external central continuum, for example by fast shocks or cosmic rays. The morphological connection between the radio and line emission suggests that the jet outflow shapes the NLR. Estimates of high gas temperatures (Kriss et al. 1992b) and the existence of dense but highly ionized clouds near the nucleus on both sides of the jet axis suggest that the jet may also play a role in photoionizing the clouds (Capetti et al 1997; Axon et al. 1998; Dietrich \& Wagner 1998). Hot stars are a third ionization mechanism. Unresolved UV continuum point sources in the inner $7^{\prime \prime} \times 7^{\prime \prime}$, which are not observed in [O III $] \lambda 5007$, could be OB associations (Macchetto et al. 1994). Hot stars are certainly a component in the ring-like structure that surrounds the nucleus. An ellipse of $\mathrm{HI}$ in emission delineates the NLR at an average radius of $13^{\prime \prime}$ (Cecil, Bland \& Tully 1978; Bruhweiler et al. 1991) and starburst knots and CO emission encircle the nucleus and the NLR at an average radius of $18^{\prime \prime}$ (Planesas, Scoville \& Myers 1991). However, the overall similarity in the profiles of the mid-infrared high-excitation lines, which cannot be excited by hot stars, and the profiles of the intermediate excitation lines $\left(E_{\text {ion }}>30 \mathrm{eV}\right)$, which could be excited by hot stars, strongly suggests that gas excited by hot stars within the large ISO aperture does not contribute more than $\sim 20 \%$ to these NLR line fluxes (Lutz et al. 2000). An additional complication due to stars is contamination of the observed emission line spectrum with stellar absorption features. This appears in the difference spectrum of the $30^{\prime \prime} \oslash$ and $18^{\prime \prime} \oslash$ HUT apertures, which shows both a reddened early-type stellar continuum and stellar absorption features (Kriss et al. 1992b).

As is discussed by Lutz et al. (2000), the ISO-SWS line ratios indicate that the mid-IR lines are emitted from gas with a hydrogen density of $\sim 2000 \mathrm{~cm}^{-3}$. The NLR appears to contain also higher density gas. The density of individual knots in the high ionization core is estimated at $10^{4}$ to $3 \times 10^{4} \mathrm{~cm}^{-3}$ from $H S T$ measurements of the [S II] doublet, while the overall density $\sim 1^{\prime \prime}$ from the nucleus varies between $10^{3}$ to $4 \times 10^{3} \mathrm{~cm}^{-3}$ (Capetti et al. 1997).

The ionization parameterf also appears to vary across the NLR. The [O III $] \lambda 5007 /(\mathrm{H} \alpha+[\mathrm{N} \mathrm{II}] \lambda 6584)$ ratio (Capetti et al. 1997) traces a high excitation core $(\log U \sim-2.5)$ in the inner $1^{\prime \prime} \times 2^{\prime \prime}$ north of the nucleus, followed by a lowered ionization halo ( $\log U \sim-3.3$ ) out to $\sim 4^{\prime \prime}$ from the nucleus, and then intermediate ionization filaments

\footnotetext{
${ }^{5} U \equiv Q_{\text {ion }} / 4 \pi r^{2} n c$, where $Q_{\text {ion }}$ is the ionizing photon luminosity, $n$ is the hydrogen density at the illuminated face of the cloud, $r$ is the distance of the face of the cloud from the continuum source and $c$ is the speed of light. The local ionization parameter in the cloud falls with increasing depth due to absorption and geometrical dilution of the radiation field.
} 
$(\log U \sim-2.8)$ which extend out to the EELR.

Estimates of dust reddening in the NLR of NGC 1068 range from $E_{\mathrm{B}-\mathrm{V}}=0.07$ for the continuum (Kriss et al 1992b), to 0.20 (Marconi et al 1996), 0.40 (Shields \& Oke 1975; Neugebauer et al 1980; Ward 1987), and $E_{\mathrm{B}-\mathrm{V}}=0.52$ (Koski 1978) for the NLR. The analysis of Kraemer et al. (1998) indicates that there may be some dust mixed with gas in varying amounts. The observed line ratios in the NLR suggest that the O/N abundance is less than solar. Netzer (1997) and Netzer \& Turner (1997) interpret this as an indication of under-abundant oxygen (see also Sternberg, Genzel \& Tacconi 1994), and propose that the abundances of He:C:N:O:Ne:Mg:Si:S:Ar:Fe relative to hydrogen are $(100: 3.7: 1.1: 2.7: 1.1: 0.37: 0.35: 0.16: 0.037: 0.4) \times 10^{-4}$, respectively. Kraemer et al. (1998) interpret the line ratios as showing an overabundance of nitrogen, as well as hinting at higher than solar iron and neon, and propose abundance ratios of $(100: 3.4: 3.6: 6.8: 2.2: 0.33: 0.31: 0.15: 0.037: 0.8) \times 10^{-4}$.

Finally, Kraemer et al. (1998) model the NLR emission with a multi-component gas model, and suggest that the low ionization emission lines are emitted by gas that is partially screened by a dense, optically thin component. They use their models to estimate that the filling factor is $F \sim 10^{-4}$.

\subsection{The ionizing continuum}

Unlike the situation in Seyfert 1 galaxies, where it is possible to observe the intrinsic SED outside the obscured range, the intrinsic SED of NGC 1068 cannot be directly observed even in the optical or X-ray bands. Only a small fraction of the AGN light is scattered into the line of sight, and can be observed against the host galaxy in polarized light. Pier et al (1994) compiled various continuum measurements in the optical, UV and X-ray, and carefully took account of aperture differences, star-light contamination, reflection by dust and bremsstrahlung emission from the scattering plasma. They conclude that the resulting reflected SED is broadly similar to that observed in Seyfert 1 galaxies. Pier et al (1994) also list various estimates of the fraction of light reflected by the scatterer. These values range from $f_{\text {refl }}=10^{-3}$ (Bland-Hawthorn \& Voit 1993) to 0.05 (Bland-Hawthorn, Sokoloski \& Cecil 1991). Pier et al (1994) argue that the most reliable estimate is $f_{\text {refl }} \sim 0.01$ to within a factor of a few. Because the reflectors are more than a hundred light years away from the continuum source (Miller et al. 1990), short-term continuum variability is unlikely to affect the reconstruction of the reflected SED.

We adopt the Pier et al. nuclear continuum SED in the UV and X-ray as the template SED (Fig. 1), and further extend it from $10 \mathrm{keV}$ to $100 \mathrm{keV}$ with a slope of $F_{\nu} \propto \nu^{-1}$. We enumerate on the unobserved UV to soft X-ray range to find the best fitting SED. 


\section{The observed line flux compilation}

In addition to the ISO-SWS mid-IR lines fluxes presented in Lutz et al. (2000), we compiled a list of UV to IR lines from the literature. The compilation initially included a list of $\sim 120$ measured line fluxes, which were obtained over the last $\sim 30$ years using various instruments with different apertures, spectral resolutions and reduction techniques. The observed lines were taken from the following references, listed by spectral band with the aperture used (where given): UV lines from Kriss et al (1992b) $\left(18^{\prime \prime} \oslash\right)$; optical lines from Osterbrock \& Parker (1965) (trailing long slit), Anderson (1970) $\left(8^{\prime \prime} \times 8^{\prime \prime}\right)$, Wampler (1971) $\left(10^{\prime \prime} \oslash\right)$, Koski (1978) $\left(2.7^{\prime \prime} \times 3.4^{\prime \prime}\right)$ and Neugebauer et al $(\overline{1980})\left(\sim 10^{\prime \prime} \times 20^{\prime \prime}\right)$; optical to near-IR lines from Shields \& Oke (1975) $\left(10^{\prime \prime} \times 10^{\prime \prime}\right)$, near-IR lines from Oliva \& Moorwood (1990) $\left(6^{\prime \prime} \times 6^{\prime \prime}\right)$, Marconi et al (1996) (long slit of width $\left.4.4^{\prime \prime}\right)$, Osterbrock \& Fulbright (1996) $\left(3^{\prime \prime} \times 18^{\prime \prime}\right)$ and Osterbrock, Tran \& Veilleux (1992) (long slit of width 1.2" $)$; IR lines from Thompson $(\overline{1996})\left(2^{\prime \prime} \times 10^{\prime \prime}\right)$ and the ISO-SWS $\left(14^{\prime \prime} \times 20^{\prime \prime}\right.$ to $\left.20^{\prime \prime} \times 33^{\prime \prime}\right)$.

As is discussed in detail by Alexander et al. (1999), the result of such a compilation is generally not self-consistent, and only a small subset of the of the $\sim 120$ lines can be used. First, we excluded low and medium excitation lines $\left(E_{\text {ion }} \lesssim 100 \mathrm{eV}\right)$ observed through apertures whose smaller dimension is less than $3^{\prime \prime}$, so as to avoid significant loss of light due to incomplete coverage of the line emitting region. The observed emission from the high excitation lines is centrally concentrated in the inner $<4^{\prime \prime}$ (Marconi et al 1996), which are covered even by the smallest apertures used. Second, we excluded lines with $E_{\text {ion }}<30 \mathrm{eV}$ to avoid using lines that may be photoionized primarily by young hot stars or other non-AGN, lower-energy excitation processes. This also reduces the loss of light bias. Third, we excluded narrow lines whose measured flux is uncertain, either because the flux is very low (flux less than $5 \times 10^{-13} \mathrm{erg} \mathrm{s}^{-1} \mathrm{~cm}^{-2}, \sim 2 \%$ of the strongest line), or because it has a significant broad component, such as the $\mathrm{N} v \lambda 1240$ and $\mathrm{C}$ IV $\lambda 1549$ lines. Fourth, we excluded the $[\mathrm{Fe} \mathrm{X}] \lambda 6734$ and $[\mathrm{Fe} \mathrm{XI}] \lambda 7892$ lines, whose collision strengths are highly uncertain and therefore cannot be modeled reliably. The final, much reduced line list includes 22 lines (Table 1), which we use for obtaining the best-fit SED. Whenever more than one measurement of the line exists, we quote the average flux and use the rms scatter as an error estimate. Important IR lines that were not included in the final list were nevertheless compared to the best fit model predictions to verify that there are no gross inconsistencies.

\section{The photoionization models}

The method of constructing photoionization models for the NLR and, in particular, the " $\log ^{2} S$ fit procedure" for obtaining the best fitting SED and gas parameters is described in detail in Alexander et al. (1999), and is summarized here briefly. We parameterize the SED as a piece-wise broken power-law (Fig. 1), and enumerate on the different possibilities of connecting the power-law segments. We test a large number of simplified NLR gas models, which consist of 
optically thick (radiation bounded) clouds whose ionized surfaces partially cover a spherical shell around the continuum source. Each cloud extends in the radial direction as far as it takes to effectively absorb all the ionizing UV photons (specifically, until the hydrogen ionization fraction falls below 2\%). The gas clouds are parameterized by their chemical composition, the hydrogen density $n$, the ionization parameter $U$ at the irradiated face of the cloud, and the filling factor $F$. In addition, an asymmetry parameter $A$ expresses the ratio between the ionizing flux directed towards the NLR and that directed towards the observer. This describes situations where the continuum source is not isotropic, or where only a fraction $f_{\text {refl }}$ of the continuum is reflected towards the observer $\left(A=1 / f_{\text {refl }}\right)$.

For each NLR gas model, the fit procedure uses the observed line fluxes to derive the best-fit SED for that gas model, and from it to derive in a self-consistent way the corresponding covering factor $C$, the inner NLR angular radius $\theta$, the width of the ionized region $\Delta \theta_{\text {ion }}$ (defined here as the radial extent of the Balmer lines emitting gas) and the reddening coefficient $E_{\mathrm{B}-\mathrm{V}}$. These parameters are constrained by the observations and so can be used to limit the range of acceptable NLR gas models. The $\log ^{2} S$ fit procedure assigns a score $S$ to the best-fit model, which means that the model line fluxes fit the observed ones up to a factor $S$, on average. The worst-fitting line and the factor by which it deviates from the observed value are also recorded. Monte-Carlo simulations are used to calculate confidence limits on the best-fit SED. In addition, we calculate the correlation between the model-to-data line ratios and the lines wavelengths, ionization potentials, critical densities and "deplitivity" (the tendency of an element to be depleted into dust). These residual correlations test whether the remaining inconsistencies in the best-fit model are related to inaccurate modeling of the reddening, the spectral hardness / softness of the continuum, the gas density or its dust content. The correct model should not display any such correlations. The final, global best-fit SED is the one with the best $S$-score among all the NLR gas models that are consistent with the observed NLR geometry and reddening, whose worst-fitting line is not too far from the observed value, and which display no significant residual correlations.

We assume in all models that the gas clouds have constant density and that $f_{\text {refl }}=0.005$. We investigate two classes of models. The first consists of models with a single type of cloud. We enumerate on different values for the ionization parameter $(\log U=-1,-2$ and -3$)$, gas density ( $n=2000$ and $10^{4} \mathrm{~cm}^{-3}$ ) and filling factor ( $\log F=-2,-3$ and -4 at the ionized surface). We test two different sets of non-solar abundances, the low oxygen set and high nitrogen set ( $\S 2.2$ ). We test two different possibilities for the radial run of the filling factor. The first is that $F$ is constant, which corresponds to either a static distribution of clouds, a rotating distribution of clouds, a linear constant velocity outflow, a strongly decelerating outflow at a constant opening angle, or an outflow where clouds are continuously added to the flow (e.g. from the molecular torus). The second is $F \propto r^{-2}$, which corresponds to a constant velocity and constant opening angle outflow where the clouds are formed at the base of the flow. The second class of models have two types of gas clouds, and are constructed by combining all the possible pairs of one component models. We assume that the clouds do not obscure one another. 
The photoionization calculations were carried out using the numerical photoionization code ION99, the 1999 version of the code ION described by Netzer (1996).

\section{Results}

\subsection{Single component models}

We find that single component models generally fail to fit the observed line ratios and the observed constraints on the geometry of the NLR. Low $U$ models $(\log U=-3)$ cannot reproduce the high excitation lines regardless of the values of the other model parameters. For the $F \propto r^{-2}$ models, the low $U$ models significantly over-estimate the size of the NLR, while the high- $U$ models require unphysical covering factors exceeding unity. Single component models with a high $U$ and low constant filling factor do somewhat better, although such models are problematic because a constant filling factor is inconsistent with simple scenarios of NLR outflow. The best fit single component model has low oxygen abundance with $\log U=-1, \log n=3.3$ and $\log F=-3$. This model can reproduce the observed lines up to a factor of 2 and predicts $\theta=0.8^{\prime \prime}, \Delta \theta_{\text {ion }}=4.8^{\prime \prime}$, $C=0.35$ and $E_{\mathrm{B}-\mathrm{V}}=0.18$. These values are roughly consistent with the observed constraints. However, this model under-predicts the observed O vi $\lambda 1035$ flux by a factor of $\sim 5$ and shows a residual negative correlation with $\lambda_{0}$ at the $5 \%$ confidence level. Like all the models investigated here, the best fit SED displays a deep trough at 4 Ryd $(\log f=-27.4,-29.0,-27.4,-28.2$ at 2, 4, 8 and 16 Ryd, respectively). A fit of similar quality is obtained with the high nitrogen abundance set.

\subsection{Two component models}

A better fit to the observations is provided by the best-fit two component model (Table 2). This model fits the 22 observed line fluxes to within a factor of 1.9 on average (experience shows this is as well as one can expect for AGN photoionization models). The worst fitting line, $[\mathrm{Ar} \mathrm{VI}] \lambda 4.5 \mu \mathrm{m}$, is under-predicted by a factor of 4 . The model-to-data ratios of individual lines are displayed in Fig. 3. The low excitation IR lines $\left(E_{\text {ion }}<30 \mathrm{eV}\right)$ such as [Ne II $] \lambda 12.8 \mu \mathrm{m}$, [S III] $\lambda 18.7 \mu \mathrm{m}$ and [S III] $\lambda 33.5 \mu \mathrm{m}$, which were not used in the fit, are nevertheless all consistent with the observations to within a factor of 2 . However, the model-to-data line ratios for these lines are not much smaller than 1 , as would be expected if there is a significant contamination from gas photoionized by starbursts. The high excitation IR lines such as [S IX] $\lambda 1.25 \mu \mathrm{m},[\mathrm{Six}] \lambda 1.4 \mu \mathrm{m}$ and [Si IX] $\lambda 2.6 \mu \mathrm{m}$, which were not used in the fit, are also consistent with the observations to within a factor of 3. There are no statistically significant residual correlations between these ratios and $\lambda_{0}, E_{\text {ion }}$, the deplitivity or $n_{c}$. The best fit values of the extinction $\left(E_{\mathrm{B}-\mathrm{V}} \sim 0.2\right)$ is in agreement with other estimates $(\oint 2.2)$. The covering factors are somewhat larger than is indicated by the observed opening angle of the emission cone (\$2.1). The dense compact component requires a 
covering factor of $C=0.45$, which corresponds to a bi-cone with an opening angle of $\sim 115^{\circ}$. The less dense, more extended component requires a covering factor $C=0.26$, which corresponds to a single cone with an opening angle of $\sim 130^{\circ}$ or a bi-cone with opening angle of $\sim 90^{\circ}$. Figure 2 shows the best fit SED for this gas model together with the $99.9 \%$ confidence limits on it (the lower confidence limits at 2, 4 and 16 Ryd were not calculated as they extend beyond the SED grid). This SED shows the generic trough that is seen in the best-fit SED of all the models we investigated. This model has a less than solar oxygen abundance (Netzer 1997; Netzer \& Turner 1997). We find only a few two-component models with high nitrogen abundance that come close to a reasonable fit to the observations. Of these, almost all display a negative correlation with deplitivity at the 5\% to $10 \%$ significance, which may indicate that the line emitting gas is more depleted than is assumed by the high nitrogen abundance set.

We conclude that best-fit two component model provides a better, but not an overwhelmingly better fit to the observed line ratios than the single component model. Its marked advantage over the single component models lies in its consistency with the observed NLR geometry and kinematics. This is further discussed in the next section.

\section{Discussion}

As was discussed in detail by Alexander et al. (1999), there are various degeneracies between the parameters that describe the gas model $\left(n, A, F, U, C, \theta\right.$, and $\left.\Delta \theta_{\text {ion }}\right)$. These degeneracies allow the fit procedure to converge to a robust best-fit SED even when the assumed values of $n$, $A, F$ or $U$ significantly differ from their true values, since this can be compensated to a large degree by a suitable modification of the gas geometry $\left(C, \theta\right.$, and $\left.\Delta \theta_{\text {ion }}\right)$. This property of the fit procedure is especially important in the analysis of NGC 1068, where observations indicate that the actual properties of the NLR gas are much more complex than can be modeled by our family of simplified gas models. For this reason, we place more weight on the fact that all the best-fit SED models, whether one or two-component, display a deep trough at 4 Ryd than on the determination of the exact values of the gas parameters.

The trough in the SED is required for reproducing the relative line fluxes of the high and low ionization species. To check the robustness of this result, we attempted to re-fit the observed line fluxes with all of our one and two-component models using an approximate single power-law SED $(\log f=-25.8,-26.6,-27.4,-28.2$ at $2,4,8$ and 16 Ryd, respectively), which was held fixed in the fit procedure. In all cases the low excitation lines were over-estimated with respect to the high excitation lines, regardless of the values of $U, n$ or $F$. For example, when the power-law SED is applied to the best-fit two-component gas model (Table 2), the low excitation lines $\left(E_{\text {ion }} \lesssim 50 \mathrm{eV}\right.$ ) are over-estimated by the model by up to a factor of 13 , whereas the high excitation lines $\left(E_{\text {ion }} \gtrsim 100 \mathrm{eV}\right)$ are under-estimated by up to a factor of 23 . The overall mismatch of this SED with the observed line fluxes is reflected in both the poor fit score of $S=4.1$ and in the very strong residual anti-correlation between the line ratios and $E_{\text {ion }}$, whose random probability is 
$10^{-4}$. We caution against generalizing this result to mean that every AGN that exhibits a hard emission line spectrum has an absorbed ionizing SED. The emission line spectrum reflects the gas parameters, such as $U, n$ and $F$, no less than it does the ionizing SED. It is necessary to have some knowledge of the likely range of values for these parameters in order to interpret the hardness of the line spectrum. Alexander et al. (1999) provide a counter-example where subtle cancellations between the SED and the gas properties lead to a situation where an AGN (NGC4151) with a hard absorbed SED has a softer emission line spectrum than another AGN (Circinus) with a soft unabsorbed Big Blue Bump.

Although we do not claim to fix the gas parameters with certainty, the best fit model (Table 2) is reassuringly consistent with the observations, which broadly indicate that the integrated NLR emission originates in two components. Component $\mathrm{A}$ of the model can be interpreted as a system of dense $\left(n=10^{4} \mathrm{~cm}^{-3}\right)$, centrally concentrated $\left(0.3^{\prime \prime}<\theta<1.4^{\prime \prime}\right)$ outflowing gas clouds $\left(F \propto r^{-2}\right)$ with a relatively high filling factor $(F \sim 0.01)$ and high ionization parameter $(\log U=-1)$. Component $\mathrm{B}$ of the model can be interpreted as a more extended distribution $\left(1.9^{\prime \prime}<\theta<4.5^{\prime \prime}\right)$ of lower density gas $\left(n=2 \times 10^{3} \mathrm{~cm}^{-3}\right)$ with no net outflow $(F=$ const. $)$, with a lower filling factor $(F=0.001)$ and a lower ionization parameter $(\log U=-2)$. Component A contributes $58 \%$ of the total line flux in 22 lines listed in Table 1, with the contribution to individual lines ranging from $45 \%$ of the low excitation $[\mathrm{S} \mathrm{IV}] \lambda 10.5 \mu \mathrm{m}\left(E_{\text {ion }}=34.8 \mathrm{eV}\right)$ to more than $99.9 \%$ of the very high excitation line $[\mathrm{Si} \mathrm{IX}] \lambda 3.9 \mu \mathrm{m}\left(E_{\text {ion }}=303.2 \mathrm{eV}\right)$. Its large covering factor indicates that there is probably a significant contribution of flux from the inner $\sim 1^{\prime \prime}$ of the diffuse $\mathrm{SW}$ emission cone as well as from the bright NE cone. Component B contributes the remaining $42 \%$ of the total line flux, mainly in the lower excitation lines. Its covering factor is small enough for it to be concentrated mostly in the NE bright emission cone, as is observed.

The best-fit procedure indicates that models with the low oxygen abundance set fit the observed line fluxes somewhat better than models with the high nitrogen abundance set. In particular, the $\mathrm{O} I I I] \lambda 1663$, whose unusual relative weakness was an important argument for assuming non-solar abundances (Netzer 1997; Kraemer et al. 1998), is well reproduced by the best-fit low oxygen model with a model-to-data line flux ratio of 1.4 (Fig. 3), even though it was not included in our fit since it didn't pass the minimal flux criterion.

The trough in the best fit SED (Fig. 2) can be interpreted as an absorption trough due to an absorber between the continuum source and the NLR. The energy resolution of the SED template, which is limited by the computational cost of enumerating over all the SED combinations, is too low to allow detailed modeling of the absorber. Figure 1 shows an example of how a quasi-thermal big blue bump that is absorbed by neutral hydrogen would appear in our low resolution SED reconstruction. We find that the trough is consistent, for example, with an absorber that shadows the entire $\operatorname{NLR}\left(C_{\mathrm{abs}}=1\right)$ and has a column density of $N_{H^{0}}=6 \times 10^{19} \mathrm{~cm}^{-2}$ in neutral hydrogen, or with an absorber that allows a small leakage of unfiltered radiation $\left(C_{\text {abs }}=0.999\right)$ and a column density of $N_{H^{0}}=10^{20} \mathrm{~cm}^{-2}$. A similar trough, consistent with an absorber of $C_{\text {abs }}>0.99$ and $N_{H^{0}}=5 \times 10^{19} \mathrm{~cm}^{-2}$, was discovered in the reconstructed SED of Seyfert 1 galaxy NGC 4151 
(Alexander et al. 1999). That absorber was also detected in the HUT absorption line spectra of the UV continuum of NGC 4151 (Kriss et al. 1992a, 1995) . The HUT spectra of NGC 1068 (Kriss et al 1992b) do not have a high enough $\mathrm{S} / \mathrm{N}$ to allow the detection of absorption lines in the scattered UV continuum of this AGN or against the stellar background (G. Kriss, private comm.). We predict that future sensitive absorption line studies should reveal the presence of such an absorber.

The bias in our results due to the fact that we neglected the line emission from the absorbing gas is likely to be small if the absorber is similar to the dense, high velocity UV absorber that was detected in NGC 4151. Such an absorber will not emit forbidden lines, and its permitted lines will be broader than typical NLR lines. Only 3 of the 22 lines we used in our fit are permitted lines, and we did not use lines that are contaminated by broad components. A highly ionized and optically thin absorber will produce strong O VI $\lambda 1035$ line emission in excess of the typical NLR emission. It is therefore interesting that unlike the two forbidden [O III $] \lambda 5007$ and [O IV] $\lambda 25.9 \mu \mathrm{m}$ lines and the semi-forbidden $\mathrm{O}$ III] $\lambda 1663$ line, which are well reproduced by the best fit model, the observed O Vi $\lambda 1035$ line is 3.2 stronger than predicted (Fig. 3).

We have, up to now, applied our SED reconstruction method to ISO-SWS observations of IR coronal lines of three AGN: the Seyfert 2 Circinus galaxy (Moorwood et al. 1996; Alexander et al. 1999), the Seyfert 1 galaxy NGC 4151 (Alexander et al. 1999), and the Seyfert 2 galaxy NGC 1068 (this work). In one of these (Circinus), we detect a Big Blue Bump that peaks at $\gtrsim 50 \mathrm{eV}$. In the other two we detect deep troughs, which are consistent (but not exclusively so) with a Big Blue Bump that is absorbed by neutral gas interior to the NLR. Our findings thus far are consistent with the picture that luminous Seyfert galaxies are powered by thin accretion disks that produce a quasi-thermal Big Blue Bump, and that in a large fraction of them the NLR sees a partially absorbed ionizing continuum, as suggested by Kraemer et al. (1998).

This work was supported by DARA under grants 50-QI-8610-8 and 50-QI-9492-3, and by the German-Israeli Foundation under grant I-0551-186.07/97.

\section{REFERENCES}

Alexander T., Sturm E., Lutz D., Sternberg A., Netzer H., \& Genzel R., 1999, ApJ, 512, 204

Alloin D., Pelat D., Boksenberg A., \& Sargent W. L. W., 1983, ApJ, 275, 493

Anderson, K. S., 1970, ApJ, 162, 743

Antonucci R., 1993, ARA\&A, 31, 473

Antonucci R. R. J., Miller J. S., 1985, ApJ, 297, 621 
Axon D. J., Marconi A., Capetti A., Macchetto F. D., Schreier E., \& Robinson A., 1998, ApJ, 496, L75

Bland-Hawthorn J., Sokoloski J., \& Cecil G., 1991, ApJ, 375, 78

Bland-Hawthorn J., \& Voit G. M., 1993, Rev, Mexicana Astron. Af., 27, 73

Bruhweiler F. C., Truong K. Q. \& Altner B., 1991, ApJ, 379, 596

Capetti A., Axon D. J., \& Macchetto F. D. Sparks W. B., Boksenberg A., 1995, ApJ, 446, 155

Capetti A., Axon D. J., \& Macchetto F. D. Sparks W. B., Boksenberg A., 1996, ApJ, 469, 554

Capetti A., Axon D. J., \& Macchetto F. D., 1997, ApJ, 487, 560

Cecil G., Bland J., Tully R. B., 1990, ApJ, 355, 70

Dietrich M., Wagner S. J., 1998, A\&A, 338, 405

Evans I. N., Ford H. C., Kinney A. L., Antonucci R. R. J., Armus L., Caganoff S., 1991, ApJ, 369, L27

George I. M.,Turner, T. J., Netzer H., Nandra K., Mushotzky R. F., \& Yaqoob T., 1998, ApJS, 114,73

Kishimoto M., 1999, ApJ, 518, 676

Koski A. T., 1978, ApJ, 223, 56

Kraemer S. B., Ruiz J. R., \& Crenshaw D. M., 1998, ApJ, 508, 232

Kraemer S. B., Turner T. J., Crenshaw D. M., \& George I. M., 1999, ApJ, in press

Kriss G. A., et al., 1992a, ApJ, 392, 485

Kriss G. A., Davidsen A. F., Blair W. P., Ferguson H. C., \& Long K. S., 1992b, ApJ, 394, L37

Kriss G. A., Davidsen A. F., Zheng W., Kruk J. W., \& Espey B. R., 1995, ApJ, 454, L7

Lipovetsky V. A., Neizvestny S. I., \& Neizvestnaya O. M., 1988, Soobshch. SAO., 55, 5

Lutz D., et al., 2000, ApJ, in preparation

Macchetto F., Capetti A., Sparks W. B., Axon D. J., \& Boksenberg A., 1994, ApJ, 435, L15

Marconi A., van der Werf P. P., Moorwood A. F. M., Oliva E., 1996, A\&A, 315, 335

Meaburn J., Pedlar A., 1986, A\&A, 159, 336

Marshall et al., 1993, ApJ, 405, 168 
Miller J. S., Goodrich R. W., \& Mathews W. G., 1991, ApJ, 378, 47

Moorwood A. F. M., et al. 1996, A\&A, 315, L109

Muxlow T. W. B., Pedlar A., Holloway A. J., Gallimore J. F., \& Antonucci R. R. J., 1996, MNRAS, 278, 854

Netzer, H., 1996, ApJ, 473, 781

Netzer, H., 1997, Ap\&SS, 248, 127

Netzer H., \& Turner T. J., 1997, ApJ, 488, 694

Neugebauer G., et al, 1980, ApJ, 283, 502

Oliva E., Moorwood A. F. M., ApJ, 1990, 348, L5

Osterbrock D. E., Fulbright J. P. , 1996, PASP, 108, 183

Osterbrock D. E., Parker R., 1965, ApJ, 141, 892

Osterbrock D. E., Tran H. D., Veilleux S., ApJ, 389, 196

Pier E. A., Antonucci R., Hurt T., Kriss G., \& Krolik J., 1994, ApJ, 428, 124

Planesas P., Scoville N. Z. \& Myers S. T., 1991, ApJ, 369, 364

Shields G. A., \& Oke J. B., 1975, ApJ, 197, 5

Shakura N. I., \& Sunyaev R. A., 1973, A\&A, 24, 337

Snijders M. A. J., Netzer H., \& Boksenberg A., 1986, MNRAS, 222, 549

Sternberg A., Genzel R., \& Tacconi L., 1994, ApJ, 436, L131

Tacconi L. J., Genzel R., Blietz M., Cameron M., Harris A. I., \& Madden S., 1994, ApJ, 426, 77

Thompson R. I., 1996, ApJ, 459, L61

Unger S. W., Lewis J. R., Pedlar A., \& Axon D. J., 1992, MNRAS, 258, 371

Wampler E. J., 1971, ApJ, 164, 1

Ward et al M. J., 1987, ApJ, 316, 138

Wilson A. S., 1988, Advances in Space Research, 8, 27

Wilson A. S., Ulvestad J, S., 1983, ApJ, 275, 8 
Fig. 1. - The template used for enumerating on the SED of NGC 1068. The most and least luminous SEDs are indicated by the lines, together with the relative sense of their spectral hardness between 1 and 150 Ryd.

Fig. 2. - The best fit SED of the two-component model (Table 2).

Fig. 3. - The model to data line ratios for the best fit two-component model (Table 2). The O III] $\lambda 1663$ line ratio (white circle), which was not used in the fitting procedure is also displayed.

Fig. 4.- An example of how a quasi-thermal Big Blue Bump that is absorbed by neutral hydrogen would appear in the low resolution reconstructed SED. The unabsorbed bump (thin line) and the bump after absorption by a $C_{\text {abs }}=1, N_{H^{0}}=6 \times 10^{19} \mathrm{~cm}^{-2}$ absorber (long dashed line) and a $C_{\text {abs }}=0.999, N_{H^{0}}=10^{20} \mathrm{~cm}^{-2}$ absorber (short dashed line) are superimposed on the best fit SED model. 
Table 1. The compiled emission line flux list.

\begin{tabular}{|c|c|c|c|c|}
\hline Line & $\begin{array}{c}\lambda_{0} \\
\mu \mathrm{m}\end{array}$ & $\begin{array}{l}E_{\text {ion }^{\mathrm{a}}} \\
\mathrm{eV}\end{array}$ & $\begin{array}{c}f_{\ell}^{\mathrm{b}} \\
10^{-13} \mathrm{er}\end{array}$ & $\begin{array}{c}\Delta f_{\ell}^{\mathrm{c}} \\
\mathrm{g} \mathrm{s}^{-1} \mathrm{~cm}^{-2}\end{array}$ \\
\hline \multicolumn{5}{|c|}{ UV, optical and NIR lines } \\
\hline O vi & $0.1032+1038$ & 113.9 & 37.4 & 3.1 \\
\hline N IV] & 0.1487 & 47.4 & 5.1 & 1.1 \\
\hline He II & 0.1640 & 54.4 & 17.7 & 5.2 \\
\hline$[\mathrm{Ne} \mathrm{V}]$ & 0.3426 & 97.1 & 15.7 & 6.7 \\
\hline$[\mathrm{Ne}$ III $]$ & $0.3868+3969$ & 41.0 & 19.2 & 2.6 \\
\hline He II & 0.4686 & 54.4 & 6.15 & 1.50 \\
\hline [O III $]$ & $0.4959+5007$ & 35.1 & 256. & 27. \\
\hline$[\mathrm{Si} \mathrm{VI}]$ & 1.96 & 166.8 & 7.5 & 0.5 \\
\hline [Si VII $]$ & 2.48 & 205.1 & 8.3 & 0.9 \\
\hline \multicolumn{5}{|c|}{ ISO-SWS IR lines } \\
\hline [Mg VIII] & 3.028 & 224.9 & 11. & 1.1 \\
\hline [Si IX] & 3.936 & 303.2 & 5.0 & 0.6 \\
\hline$[\mathrm{Mg} \mathrm{IV}]$ & 4.49 & 80.1 & 7.6 & 1.5 \\
\hline$[\mathrm{Ar} \mathrm{VI}]$ & 4.528 & 75.0 & 15. & 3. \\
\hline [Mg VII] & 5.50 & 186.5 & 13. & $?$ \\
\hline$[\mathrm{Mg} \mathrm{V}]$ & 5.61 & 109.2 & 18. & 2. \\
\hline$[\mathrm{Ne} \mathrm{VI}]$ & 7.64 & 126.2 & 110. & 11. \\
\hline$[\mathrm{S} \mathrm{IV}]$ & 10.51 & 34.8 & 58. & 6. \\
\hline$[\mathrm{Ne} \mathrm{V}]$ & 14.32 & 97.1 & 97.0 & 9.7 \\
\hline [Ne III] & 15.56 & 41.0 & 160. & 32. \\
\hline$[\mathrm{Ne} \mathrm{v}]$ & 24.32 & 97.1 & 70. & 7. \\
\hline [O IV] & 25.89 & 54.9 & 190. & 20. \\
\hline [Ne III] & 36.01 & 41.0 & 17. & 3. \\
\hline
\end{tabular}

a The ionization energy required to produce the emitting ion from the preceding ionization stage.

b The observed flux. For permitted lines, the flux of the decomposed narrow component is quoted.

c The error estimate on the observed flux. The ISOSWS errors are estimated by the scatter in various methods for defining the underlying continuum and measuring the line. The errors do not include systematic calibration errors, which are generally smaller than $30 \%$. A question mark means that error estimates are unavailable. 
Table 2. The best-fit two-component model.

\begin{tabular}{|c|c|c|c|}
\hline & & Component A & Component B \\
\hline \multicolumn{4}{|l|}{ Input parameters: } \\
\hline & $U$ & 0.1 & 0.01 \\
\hline & $n$ & $10^{4} \mathrm{~cm}^{-3}$ & $2 \times 10^{3} \mathrm{~cm}^{-3}$ \\
\hline & Composition & low oxygen & low oxygen \\
\hline & $A$ & 200 & 200 \\
\hline & $F^{\mathrm{a}}$ & $10^{-2} h$ & $10^{-3} h$ \\
\hline & $\frac{d \log F}{d \log r}$ & -2 & 0 \\
\hline \multicolumn{4}{|l|}{ Best-fit results: $^{\mathrm{b}}$} \\
\hline & $S$ & \multicolumn{2}{|c|}{1.9} \\
\hline & $\max S_{\ell}^{c}$ & \multicolumn{2}{|c|}{$4.0^{-1}$} \\
\hline & worst line & \multicolumn{2}{|c|}{$[\mathrm{Ar} \mathrm{VI}] \lambda 4.5 \mu \mathrm{m}$} \\
\hline & $\log f_{2} \mathrm{~d}$ & \multicolumn{2}{|c|}{$-29.0(-29.0,-26.6)$} \\
\hline & $\log f_{4}$ & \multicolumn{2}{|c|}{$-29.0(-29.0,-26.6)$} \\
\hline & $\log f_{8}$ & \multicolumn{2}{|c|}{$-27.4(-27.4,-26.6)$} \\
\hline & $\log f_{16}$ & \multicolumn{2}{|c|}{$-29.0(-29.0,-28.2)$} \\
\hline & $C$ & 0.45 & 0.29 \\
\hline & $\theta$ & $0.26^{\prime \prime}$ & $1.9^{\prime \prime}$ \\
\hline & $\Delta \theta_{\text {ion }}$ & $1.1^{\prime \prime}$ & $2.6^{\prime \prime}$ \\
\hline & $E_{\mathrm{B}-\mathrm{V}}$ & \multicolumn{2}{|c|}{$0.23(0.18,0.29)$} \\
\hline & $q_{\text {ion }}$ & \multicolumn{2}{|c|}{$0.24 \mathrm{~s}^{-1} \mathrm{~cm}^{-2}$} \\
\hline & $\log Q_{\text {ion }} / \mathrm{s}^{-1}$ e & \multicolumn{2}{|c|}{54.2} \\
\hline & $\langle h \nu\rangle^{\mathrm{f}}$ & \multicolumn{2}{|c|}{ 5.1 Ryd } \\
\hline \multirow[t]{5}{*}{ Residual correlations: } & & Correlation & Random prob. \\
\hline & $\lambda_{0}$ & -0.19 & 0.23 \\
\hline & $E_{\text {ion }}$ & 0.00 & 1.00 \\
\hline & depletion & +0.11 & 0.70 \\
\hline & $n_{c}$ & +0.03 & 0.86 \\
\hline
\end{tabular}

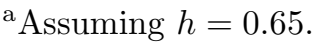

${ }^{\text {b}}$ Values in parentheses are the $99.9 \%$ confidence intervals.

${ }^{\mathrm{c}}$ Model to data ratio, given as the reciprocal when $<1$.

${ }^{\mathrm{d}}$ Flux in $\mathrm{erg} \mathrm{s}^{-1} \mathrm{~cm}^{-2} \mathrm{~Hz}^{-1}$.

${ }^{\mathrm{e}}$ Ionizing photon luminosity assuming isotopic emission.

${ }^{\mathrm{f}}$ Mean ionizing photon energy between 1 and 16 Ryd. 


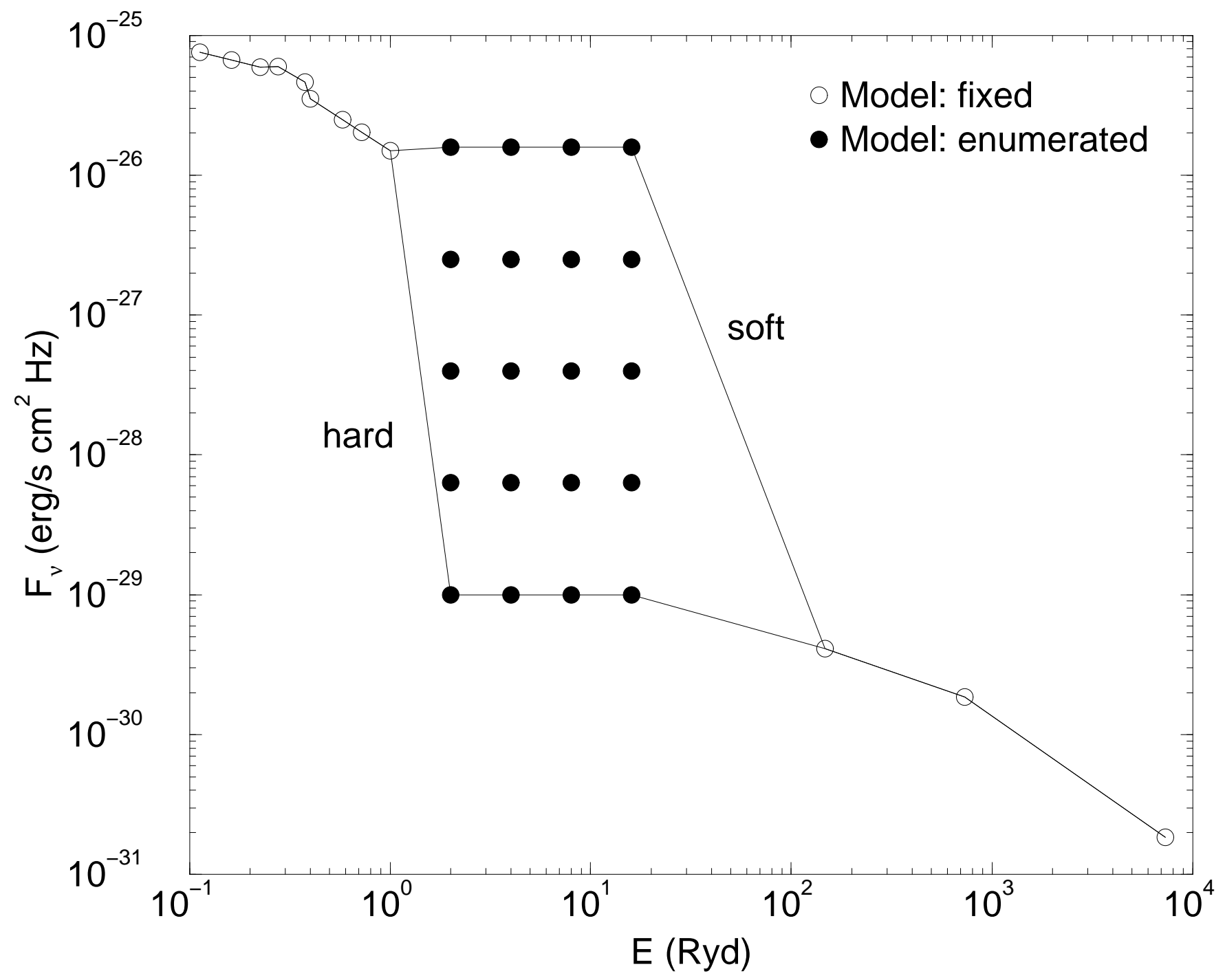




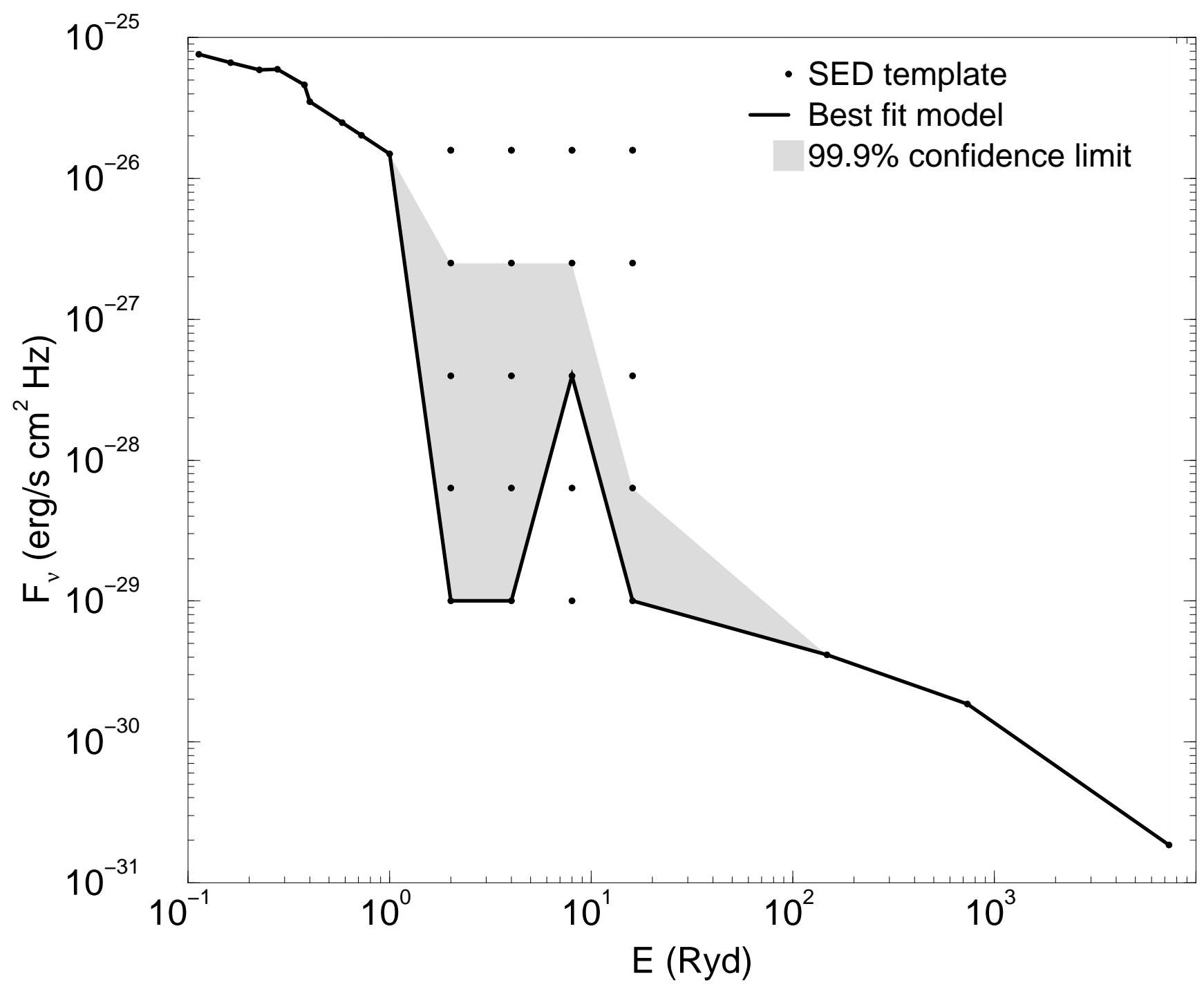




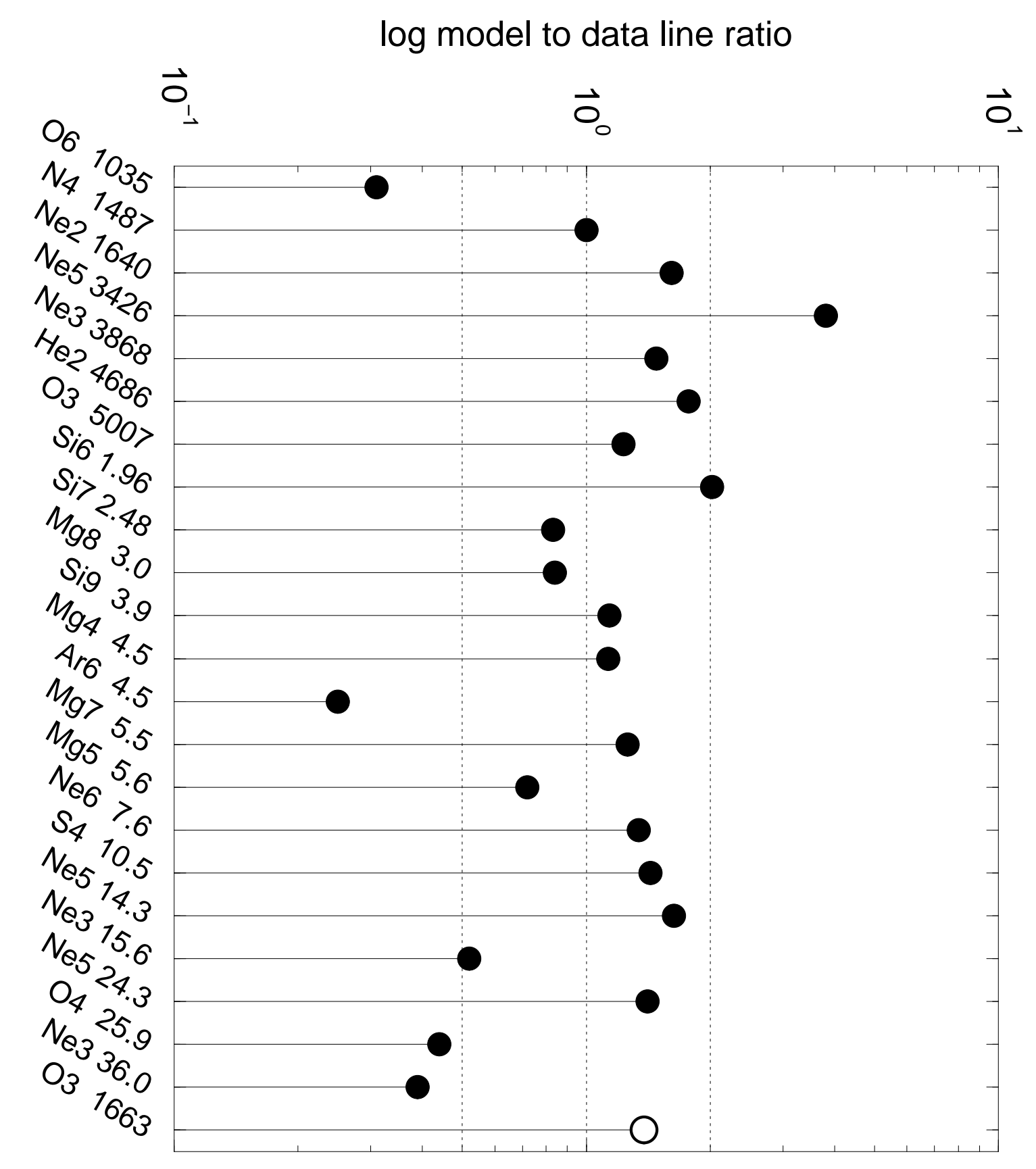




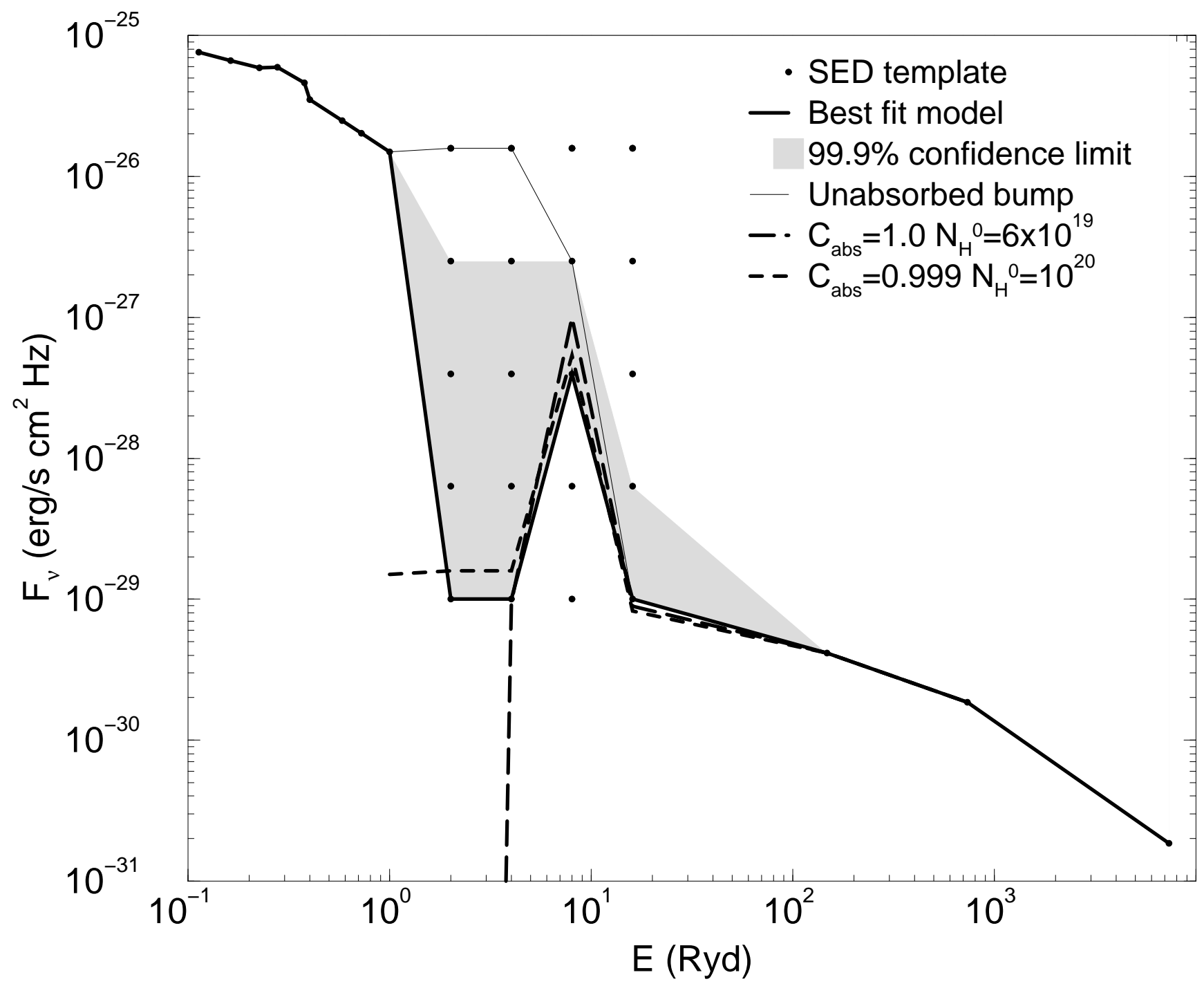

